\title{
CONDUCTANCE ANOMALIES IN STRAINED QUANTUM WIRES: THE CASE OF PbSe AND PbTe*
}

\author{
G. Grabecki, J. Wróbel, T. Diett, M. SaWicki, T. Skośkiewicz \\ Institute of Physics, Polish Academy of Sciences \\ Al. Lotników 32/46, 02-668 Warszawa, Poland \\ E. Papis, E. Kamińska, A. Piotrowska.
}

Institute of Electron Technology, Al. Lotników 32/46, 02-668 Warszawa, Poland

N. Frank, Y. Ueta, G. Sprinholtz and G. Bauer

Institüt für Halbleiterphysik, Johannes Kepler Universität Linz, 4040 Linz, Austria

We show that conducting edge channels are formed in free standing wires of $\mathrm{PbSe} / \mathrm{BaF}_{2}$ and $\mathrm{PbTe} / \mathrm{BaF}_{2}$ as temperature is lowered. The effect results from spatially inhomogeneous strain caused by a difference between the thermal expansion coefficients of the epilayer and the substrate. The presence of the edge channels can explain anomalous mesoscopic effects observed previously in these wires.

PACS numbers: $71.55 . \mathrm{Jv}$

Recent works $[1,2]$ have shown that the magnetoconductance of Hall bridges patterned from epitaxial films of $\mathrm{PbSe}$ and $\mathrm{PbTe}$ grown on a $\mathrm{BaF}_{2}$ substrate exhibits a number of novel features. In particular, the aperiodic conductance fluctuations which occur at low temperatures cannot be described within the framework of theory of universal conductance fluctuations (UCF) [3]. In particular, the fluctuation amplitude is about 10 times greater than that predicted by the theory, and the correlation function of the fluctuations gives the size of the coherence regions smaller than the mean free path for elastic scattering [1].

In order to shed some light on this phenomenon, we have undertaken new systematic studies of conductance phenomena in microstructures of both $\mathrm{PbSe}$ and $\mathrm{PbTe}$. PbSe films with (111) orientation and thickness of about $1.7 \mu \mathrm{m}$ were deposited by $\mathrm{MBE}$ directly onto the $\mathrm{BaF}_{2}$ substrate. The microstructures were fabricated by photolithography and $\mathrm{Ar}$ ion milling. $\mathrm{PbTe}: \mathrm{Bi}$ films with (111) orientation and thickness of $0.5 \mu \mathrm{m}$ were grown by $\mathrm{MBE}$ onto $\mathrm{BaF}_{2}$ substrate with $\mathrm{Pb}_{0.94} \mathrm{Eu}_{0.06} \mathrm{Te}$ buffer layer (see inset to Fig. 2). Microstructures in the form of six-terminal Hall bridges were fabricated by electron beam lithography followed

*This work was partially supported by the Committee for Scientific Research grant PBZ 101. 
by either Ar ion milling or wet etching. The initial materials were $n$-type with electron concentrations in the range $1 \times 10^{16}$ to $6 \times 10^{17} \mathrm{~cm}^{-3}$ and IIall mobilities $1.2 \times 10^{3} \mathrm{~cm}^{2} /(\mathrm{V} \mathrm{s})$ to $2 \times 10^{5} \mathrm{~cm}^{2} /(\mathrm{V} \mathrm{s})$. The widths of the wires are in the range 0.5 to $100 \mu \mathrm{m}$. The transport measurements were performed for various contact configurations $R_{i j, k l}$ (where $i j$ and $k l$ denote the current and voltage probes, respectively), as a function of temperature in the range of $30 \mathrm{mK}-300 \mathrm{~K}$.

The temperature dependence of the resistance in one of our PbSe microstructures is shown in Fig. 1 for two different probe configurations. $R_{12,34}$ represents local resistance. Its nearly linear increase with $T$ at $T>100 \mathrm{~K}$ is due to the electron-phonon scattering. Surprisingly, when the temperature is lowered below $T=50 \mathrm{~K}, R_{12,34}$ exhibits an unusual increase. At temperatures below $200 \mathrm{~K}$, the pronounced nonlocal resistance, $R_{35,46}$, is observed. It should be noted that for the uniform conductors the nonlocal resistance should be smaller than the local one by the factor $\exp (-\pi L / W)$. For the considered PbSe microstructure (see Fig. 1, inset) this formula predicts $R_{35,46}$ to be smaller by many orders of magnitude than that observed at $T<200 \mathrm{~K}$. This indicates that the cooling process leads to the occurrence of inhomogeneities within the wire.

We suggest that strain caused by a difference of thermal contraction coefficients of the wire and of the substrate material is the origin of this effect. It is

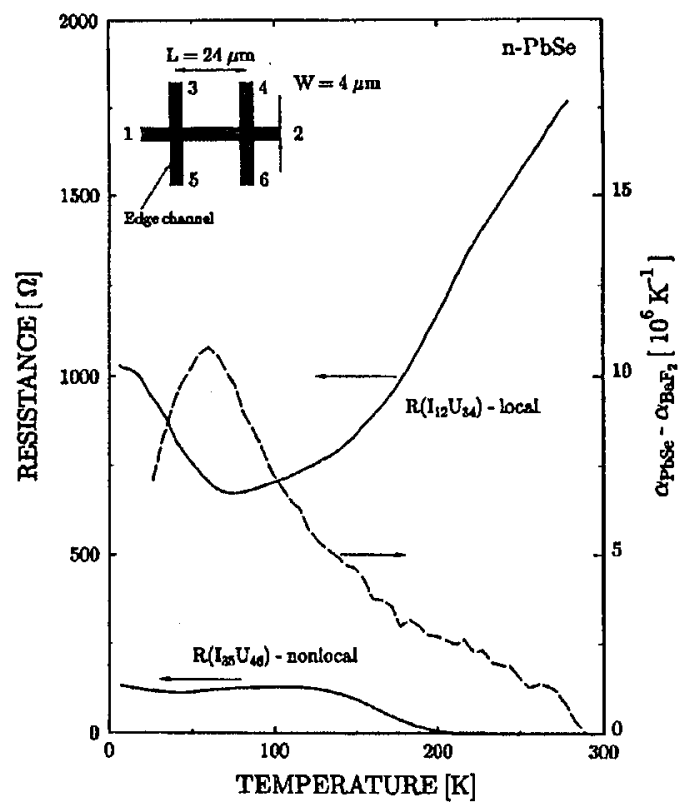

Fig. 1. Local and nonlocal resistances as a function of temperature for the $n$-PbSe quantum wire on $\mathrm{BaF}_{2}$ substrate $\left(n=6 \times 10^{16} \mathrm{~cm}^{-3}, \mu=0.12 \mathrm{~m}^{2} /(\mathrm{V} \mathrm{s})\right)$. Dashed line indicates the difference between thermal compression coefficients of the layer and the substrate materials. The inset represents the wire geometry. 
known that $\mathrm{PbTe}$ and $\mathrm{PbSe}$ epitaxial films deposited on $\mathrm{BaF}_{2}$ become elastically strained at low temperatures [4]. The difference of thermal contraction coefficients has been calculated using the data from Ref. [5] and is represented in Fig. 1 by the dashed line. Inside the layer, far from its edges, the tensile strain is uniformly biaxial and lifts the fourfold degeneracy of the $L$ states in the conduction band. In particular, the [111] valley which has its main axis oriented parallel to the growth direction is shifted downward in energy with respect to the three remaining valleys oriented with their main axes obliquely to the growth direction $[6,7]$. In the $n$-type material, this shift leads to modification of the carrier distribution between the valleys. Moreover, in the close vicinity of the edges one can expect considerable variations of the strain magnitude [8]. To explain our data, we assume that these variations lead finally to the local increase in electron concentration at the wire edges. This produces edge channels with the enhanced conductance, which give rise to the observed nonlocal effects.

The presence of edge channels is documented further by transport studies as a function of the wire width. As shown in Fig. 2, the ratio between the nonlocal and local resistances increases strongly with the decreasing wire width. Probably, in our narrowest wires, conductance is totally dominated by the edge channels. In agreement with the model, the edge effects are significantly stronger for $\mathrm{PbSe}$,

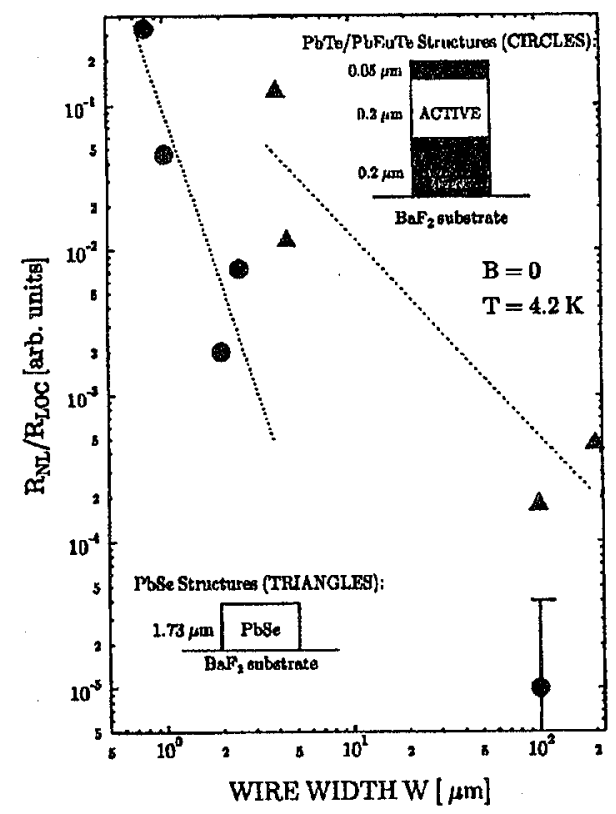

Fig. 2. The ratio between nonlocal and local resistances as a function of width for series of $\mathrm{PbSe}$ and $\mathrm{PbTe}$ wires. For $W<10 \mu \mathrm{m}$, the $\mathrm{PbTe}$ and $\mathrm{PbSe}$ wire lengths $L$ are $5.5 \mu \mathrm{m}$ and $24 \mu \mathrm{m}$, respectively. The insets represent cross-sections of the wires. 
which is directly deposited on $\mathrm{BaF}_{2}$, than in the case of $\mathrm{PbTe} / \mathrm{PbEuTe}$, where the conducting layer is separated from the substrate by the buffer layer.

Presumably, the conducting edge channels give rise to the anomalously large aperiodic magnetoconductance fluctuations observed in these wires. If the channel width is sufficiently small, it can be regarded as a one-dimensional quantum wire even if the geometrical width of the sample is much larger. This explains the observations of UCFs even in macroscopic samples with $W \approx 100 \mu \mathrm{m}$ [1]. Additionally, the electron scattering rate in a vicinity of the wire edge could be much larger than inside, possibly due to surface defects. Thus, the corresponding mean free path for electrons in the channels is much smaller than in the bulk layer. In such a case, the diffusive interfering trajectories of small size exist. This can explain unusually small coherence areas deduced from the correlation function of UCFs observed in both $\mathrm{PbTe}$ and $\mathrm{PbSe}$ wires.

\section{References}

[1] G. Grabecki, S. Takeyama, S. Adachi, Y. Takagi, T. Dietl, E. Kamińska, A. Piotrowska, E. Papis, N. Frank, G. Bauer, Jpn. J. Appl. Phys. A 34, 4433 (1995); Acta Phys. Pol. A 88, 727 (1995).

[2] J. Oswald, M. Pippan, G. Heigl, G. Span, T. Stellberger, D.K. Maude, J.C. Portal, Surf. Sci., in press and private communication.

[3] B.L. Al'tshuler, B.I. Skhlovskii, Zh. Eksp. Teor. Fiz. 91, 220 (1986); P.A. Lee, A. Douglas Stone, H. Fukuyama, Phys. Rev. B 35, 1039 (1987).

[4] H. Pasher, E.J. Fantner, G. Bauer, A. Lopez-Otero, Solid State Sciences 24, 244 (1981).

[5] J.I. Ravich, B.A. Efmowa, I.A. Smirnow, Semiconducting Lead Compounds,. Plenum, New York 1968; A.C. Bailey, B. Yates, Proc. Phys. Soc. 91, 390 (1967).

[6] J. Oswald, B.B. Goldberg, G. Bauer, P.J. Stiles, Phys. Rev. B 40, 3032 (1989); J. Oswald, P. Pichler, G. Bauer, Phys. Rev. B 49, 17029 (1994).

[7] M.V. Valeiko, I.I. Zasavitzki, A.V. Matveenko, B.N. Matsonashvili, Z.A. Rukhadze, Superlattices Microstruct. 9, 195 (1991).

[8] E. Suhir, J. Appl. Mech. 55, 143 (1988); A. Fisher, H. Richter, Appl. Phys. Lett. 61, 2656 (1992). 